\title{
A NOTE ON REICHENBACH'S AXIOMS FOR PROBABILITY IMPLICATION
}

\author{
J. C. C. McKINSEY
}

In Hans Reichenbach's book Wahrscheinlichkeitslehre, the following axioms, ${ }^{*}$ among others, are asserted for the relation of "probability implication":

I.

$$
(p \neq q) \supset\left[\left(O \rightarrow_{p} P\right) \cdot\left(O \rightarrow_{q} P\right) \equiv(\bar{O})\right] \cdot \dagger
$$

II2.

$$
\left(O \rightarrow_{p} P\right) \supset(p \geqq 0) \text {. }
$$

III. $\quad\left(O \rightarrow_{p} P\right) \cdot\left(O \rightarrow_{q} Q\right) \cdot(O \cdot P \supset \bar{Q}) \supset\left(O \rightarrow_{r} P \vee Q\right) \cdot(r=p+q)$.

The proposal is made by Reichenbach, that these axioms be added to a system of logic. The exact character of this system of logic is not specified, but we are presumably to suppose that it is something like the system of Principia Mathematica. I must refer the reader to Reichenbach's book for an explanation of the notation occurring in these axioms. Reichenbach does not explicitly state the range of variation of the variables $p, q, r$, and so on; I shall suppose he intends that these variables can assume as values any real numbers, $₫$ including also negative real numbers, and positive real numbers greater than +1 .

I shall now show that these axioms lead to a contradiction.

From Axiom I, we can easily derive the following:

$$
(\bar{O}) \supset\left(O \rightarrow_{p} P\right) .
$$

(This is stated as a theorem by Reichenbach on p. 67.) From (1) we get, by substitution,

$$
(\overline{O \cdot \bar{O}}) \supset\left(O \cdot \bar{O} \rightarrow_{p} P\right) .
$$

Reichenbach defines (on p. 67), the expression $(\bar{O})$ as follows:

$$
(\bar{O})=(i)\left(\overline{x_{i} \varepsilon}\right) .
$$

* See p. 65 and p. 69.

$\dagger$ For typographical reasons, I express the proposition " $O$ implies $P$ with probability of degree $p$ " by the symbolism " $O \rightarrow_{p} P$," instead of by the symbolism of Reichenbach.

$\ddagger$ We might, on the other hand, suppose that these variables can assume as values only real numbers from the closed interval $(0,1)$. It is not very plausible, however, to suppose this is what Reichenbach intends; such a supposition, moreover, leads in turn to difficulties. 
From (3) we have, by substitution,

$$
\left(\overline{O \cdot \bar{O})}=(i) \overline{\left(x_{i} \varepsilon O \cdot \bar{O}\right.}\right) .
$$

Since the right member of (4) is a provable sentence, the left member is provable also, and we have

$$
(\overline{O \cdot \bar{O}}) \text {. }
$$

From (5) and (2), by modus ponens, we derive

$$
\left(O \cdot \bar{O} \rightarrow_{p} P\right) .
$$

If now we replace $O$ by $O \cdot \bar{O}$ in Axiom II2, we have

$$
\left(O \cdot \bar{O} \rightarrow_{p} P\right) \supset(p \geqq 0) .
$$

From (6) and (7), by modus ponens, we have

$$
p \geqq 0 \text {. }
$$

Since it is being assumed that the variable $p$ can assume as a value any real number (and thus, in particular, a negative real number), it is seen that (8) is a contradiction.

This contradiction can be avoided by modifying Axiom I as follows:

$$
\text { I'. } \begin{aligned}
{[(p \geqq 0) \cdot(1 \geqq p) \cdot(q \geqq 0) \cdot(1 \geqq q) \cdot(p \neq q)] } & \\
& \supset\left[\left(O \rightarrow_{p} P\right) \cdot\left(O \rightarrow_{q} P\right) \equiv(\bar{O})\right] .
\end{aligned}
$$

Since similar difficulties arise in connection with Axiom III, it would be desirable also to modify this axiom as follows:

$$
\begin{aligned}
& \text { III'. }^{\prime}\left[(p \geqq 0) \cdot(q \geqq 0) \cdot(1 \geqq p+q) \cdot\left(O \rightarrow_{p} P\right) \cdot\left(O \rightarrow_{q} Q\right) \cdot(O \cdot P \supset \bar{Q})\right] \\
& \supset\left[\left(O \rightarrow{ }_{r} P \vee Q\right) \cdot(r=p+q)\right] \text {. }
\end{aligned}
$$

These changes in the axioms would, of course, entail modifying certain of Reichenbach's theorems. It would be found necessary, for example, to add as an additional hypothesis to certain theorems, conditions like $1 \geqq p$, or $q \geqq 0$, or $1 \geqq p+q$, and so on. It would also be desirable* to add to some of Reichenbach's theorems the hypothesis $\overline{(\bar{O})}$.

New York University

\footnotetext{
* This fact was called to my attention by the referee.
} 